\title{
INTRANEURONAL INJECTION OF HORSERADISH PEROXIDASE LABELS GLIAL CELLS ASSOCIATED WITH THE AXONS OF THE GIANT METACEREBRAL NEURON OF APLYSIA ${ }^{1}$
}

\author{
RONALD S. GOLDSTEIN, KLAUDIUSZ R. WEISS, AND JAMES H. SCHWARTZ ${ }^{2}$
}

Departments of Anatomy, Neurology, Physiology, and Psychiatry, Center for Neurobiology and Behavior, College of Physicians and Surgeons and School of Dental and Oral Surgery, Columbia University, New York, New York 10032

Received March 8, 1982; Revised June 1, 1982; Accepted June 2, 1982

\begin{abstract}
After injecting the cell body of the giant serotonergic neuron (GCN) in the cerebral ganglion of Aplysia with horseradish peroxidase (HRP), we found that some glial cells were labeled along the cerebrobuccal connective and posterior lip nerve, the two nerves that contain the major axon branches of the injected neuron. Starting at a distance of about $3 \mathrm{~mm}$ from the site of the injection, only glial cells in the nerve that are close to an axon branch of the GCN contained HRP. Labeling appears to be selective because, at most, only one out of three glial cells was labeled within an area of $20 \mu \mathrm{m}$ of the axon. Moreover, no HRP was detected in any of the many glial cells within the cerebral ganglion. Reaction product diffusely labeled the cytoplasm of glial cell bodies and processes after intraneuronal injection of HRP. This distribution was markedly different from that observed in glial cells after the uptake of HRP from the extracellular space; HRP, presumed to be taken up by endocytosis, was found to be localized to vesicles, tubules, and multivesicular bodies.
\end{abstract}

The ability to identify individual nerve cells with characteristic properties has been extremely useful for studying how neurons function in nervous systems (see, for example, Kandel, 1976). Like many neurons, some glial cells, such as the packet cell glia of the leech (Coggeshall and Fawcett, 1964), can be recognized as individuals from ganglion to ganglion. Other glial cells, such as astrocytes or Schwann cells, can be categorized by location or morphological appearance into general classes with distinctive functional properties (for review, see Orkand, 1977). A characteristic that could distinguish individual glial cells from others within the same general class is a special relationship with a particular identified neuron.

One special relationship that has been suggested is the transfer of molecules between glial cells and neurons (for reviews, see Kuffler and Nicholls, 1966; Varon and

\footnotetext{
${ }^{1}$ We thank Drs. Michael Gershon, Ann-Judith Silverman, Craig Bailey, Eric Kandel, Henry Kistler, and Irving Kupfermann for their critical reading of the manuscript and Eric Holtzman for his helpful suggestions. The research was supported by Grants MH35564 and MH00304 to K. R. W. and Grant NS 12066 to J. H. S. from the National Institutes of Health.

${ }^{2}$ To whom correspondence should be addressed at the Center for Neurobiology and Behavior, College of Physicians and Surgeons, Columbia University, New York, NY 10032.
}

Somjen, 1979). Giant invertebrate axons have been reported to receive newly synthesized proteins from glia (Lasek et al., 1977; Gainer et al., 1977). The unidentified materials transferred are presumed to serve a trophic function because invertebrate axons can survive for long periods after being severed from their cell bodies. Several different mechanisms of transfer have been proposed. Lasek et al. (1977) suggested that proteins are released from glia by exocytosis and locally taken up into the axon by endocytosis. In the crayfish, Perrachia (1981) has observed cytoplasmic continuities between giant axons and glial cells that would permit the passage of macromolecules, and Viancour et al. (1981) found that the dye Lucifer Yellow, when injected intra-axonally, labels the cell bodies of adaxonal glial cells.

An intriguing example of a specific interaction between an identified neuron and glial cells was described recently for the giant serotonergic metacerebral neuron of Aplysia (GCN) (Schwartz and Shkolnik, 1981). This neuron, in addition to terminating peripherally in buccal muscle (Weiss et al., 1978) and centrally on the axons and cell bodies of other neurons, also forms appositions with the morphological characteristics of synapses on glial cells. In this paper, we present further evidence that the GCN interacts specifically with some glial cells.

A preliminary report of this study has been published (Goldstein et al., 1982). 


\section{Materials and Methods}

Aplysia californica, weighing 75 to $125 \mathrm{gm}$ and supplied by Pacific Bio-Marine Laboratories, Venice, CA, were kept at $15^{\circ} \mathrm{C}$ in circulating Instant Ocean (Aquarium Systems, Mentor, OH). The cerebral and buccal ganglia were removed with the cerebrobuccal connective and posterior lip nerves attached. The tissue was pinned in a chamber filled with a supplemented artificial sea water containing antibiotics (Eisenstadt et al., 1973). Before injection of the GCN, the overlying connective sheath was removed by dissection. The completeness of the removal was later verified histologically. Using double barreled micropipettes, we injected the GCN with $4 \%$ horseradish peroxidase (HRP; type VI, Sigma Chemical Co., St. Louis, MO.) in $0.5 \mathrm{M} \mathrm{KCl}$ using pressure (Muller and McMahan, 1976). The resting potential of the neuron was monitored before, during, and after the injection; neurons were discarded if they did not have resting potentials of at least $50 \mathrm{mV}$ or if spikes could not be elicited. The injections were performed over a period of 15 to $30 \mathrm{~min}$ in order to introduce large quantities of HRP without damaging the cells. Nervous tissue was kept for 6 or $20 \mathrm{hr}$ at $15^{\circ} \mathrm{C}$ in the supplemented artificial sea water. No loss in resting potential was noted when the injected cells were impaled again after these periods of maintenance. Similar results were obtained after both the shorter and the longer period of maintenance. In four experiments, $\left[{ }^{3} \mathrm{H}\right]$ fucose was injected into a GCN using previously described methods (Thompson et al., 1976), and the tissue was kept at $15^{\circ} \mathrm{C}$ for $24 \mathrm{hr}$.

Extracellular injection of HRP. A solution of $0.15 \%$ HRP and $0.85 \%$ dextran blue (Pharmacia, Piscataway, N.J) in the supplemented sea water was pressure-injected into the neuropil of a buccal hemiganglion with a glass micropipette. The dextran blue was included to monitor the injection under the dissecting microscope. During the injection, which lasted 15 to $30 \mathrm{~min}$, the mixture could be seen flowing into the ipsilateral buccal nerves, across the buccal commissure, and down both cerebrobuccal connectives toward the cerebral ganglion. The tissue was fixed within 30 min of the injection.

Preparation of tissue for cytochemistry and microscopy. We followed the cytochemical procedures of Muller and McMahan (1976) as modified by Bailey et al. (1979) for localizing peroxidase. For conventional electron microscopic examination, tissue was fixed either in $6 \%$ glutaraldehyde (Thompson et al., 1976) or in 3\% glutaraldehyde and 3\% paraformaldehyde and then osmicated. Some tissue then was stained en bloc in $0.5 \%$ uranyl acetate in $25 \mathrm{~mm}$ Tris- $\mathrm{HCl}(\mathrm{pH} 7.6)$ and $30 \%$ sucrose. Tissue was embedded in an epoxy resin (EMbed 812; EMS, Fort Washington, PA), and sectioned at 1 to $4 \mu \mathrm{m}$ for light microscopy and at 80 to $100 \mathrm{~nm}$ for electron microscopy (determined by interference color).

\section{Results}

After injecting the cell body of the GCN, we found not only that HRP had filled the GCN's axons in the cerebrobuccal connective and posterior lip nerve but also that some of the glial cells surrounding the axon contained the label. Upon examining whole mounts of the nervous tissue after reaction with diaminobenzidine
(DAB) (Fig. 14), we observed that the axons of the injected cell were not well defined; the reaction product had a fuzzy appearance in both the connective and the posterior lip nerve. In 1- $\mu \mathrm{m}$ cross-sections viewed in the light microscope, the axon was filled homogeneously; it had a lobulated appearance due to extensive glial invagination (Fig. $1 B$ ) and was surrounded by thin processes of glial cells, some of which were filled with HRP. Labeled glial processes were seen near the axon in the nerve from its origin approximately $3 \mathrm{~mm}$ from the cell body and extending distally several millimeters along the connective. The distalmost extent of labeling depended on the time after injection. In some 20 -hr experiments, labeled glial processes were observed along the entire length of the connective. Labeled glial cells were observed only within a radius of about $20 \mu \mathrm{m}$ of the GCN's axon. Within this area, in the sections examined, at most, one out of three glial cell bodies was labeled.

Electron microscopic examination of the connective revealed that both labeled and unlabeled glial cells and processes contained small lysosomes and made typical desmosomes with labeled and unlabeled glial cells (Figs. 2 and 3). In the labeled glial cells, HRP was distributed diffusely within the cytoplasm and was excluded by mitochondria, gliosomes, and nuclei. The cytoplasm in glial cells was also characteristically more darkly stained than the axoplasm (Fig. 3). In cross-section, the processes are 0.2 to $0.5 \mu \mathrm{m}$ in diameter and appear finger-like. Staining of the glial processes by HRP allowed their serial reconstruction: they extended up to $10 \mu \mathrm{m}$ from the glial cell bodies and were over $3 \mu \mathrm{m}$ wide. Thus, the processes form plates rather than finger-like extensions as Coggeshall (1967) previously suggested.

To determine whether endogenous peroxidase activity appeared in glia as a result of the injection, in four experiments, a GCN was injected with $\left[{ }^{3} \mathrm{H}\right]$ fucose and maintained for $24 \mathrm{hr}$. Intrasomatic injection of the sugar permitted subsequent autoradiographic identification of the GCN's axon in the connective as well as serving as a control for any trauma caused by the injection procedure itself. After treatment with DAB (see "Materials and Methods"), no brown reaction product was seen in the axon of the injected cell, which was identified by autoradiography. Moreover, there was no reaction product in glial cells or in any other component of the connective in light microscopic (Fig. 1C) or electron microscopic sections (data not shown). In Figure $1 C$, silver grains from $\left[{ }^{3} \mathrm{H}\right]$ fucose were localized over the axon of the GCN and not over any other structure. Restriction of the label to the injected cell and its processes has been observed previously (Ambron et al., 1980; Schwartz and Shkolnik, 1981; Thompson et al., 1976). Presumably, the membranous organelles into which the sugar is incorporated are too large to be transferred to glial cells.

Glial cells in the ganglion are not labeled. In contrast to the glial cells in the connective, no glial cells were labeled in the ganglion containing the injected cell body. In whole mounts, the outlines of the injected GCN and its initial segment were sharply defined by reaclion product (Fig. $1 A$ ), but the axon's path within the cerebral ganglion was difficult to follow because of the opacity of the connective tissue and surrounding neural compo- 

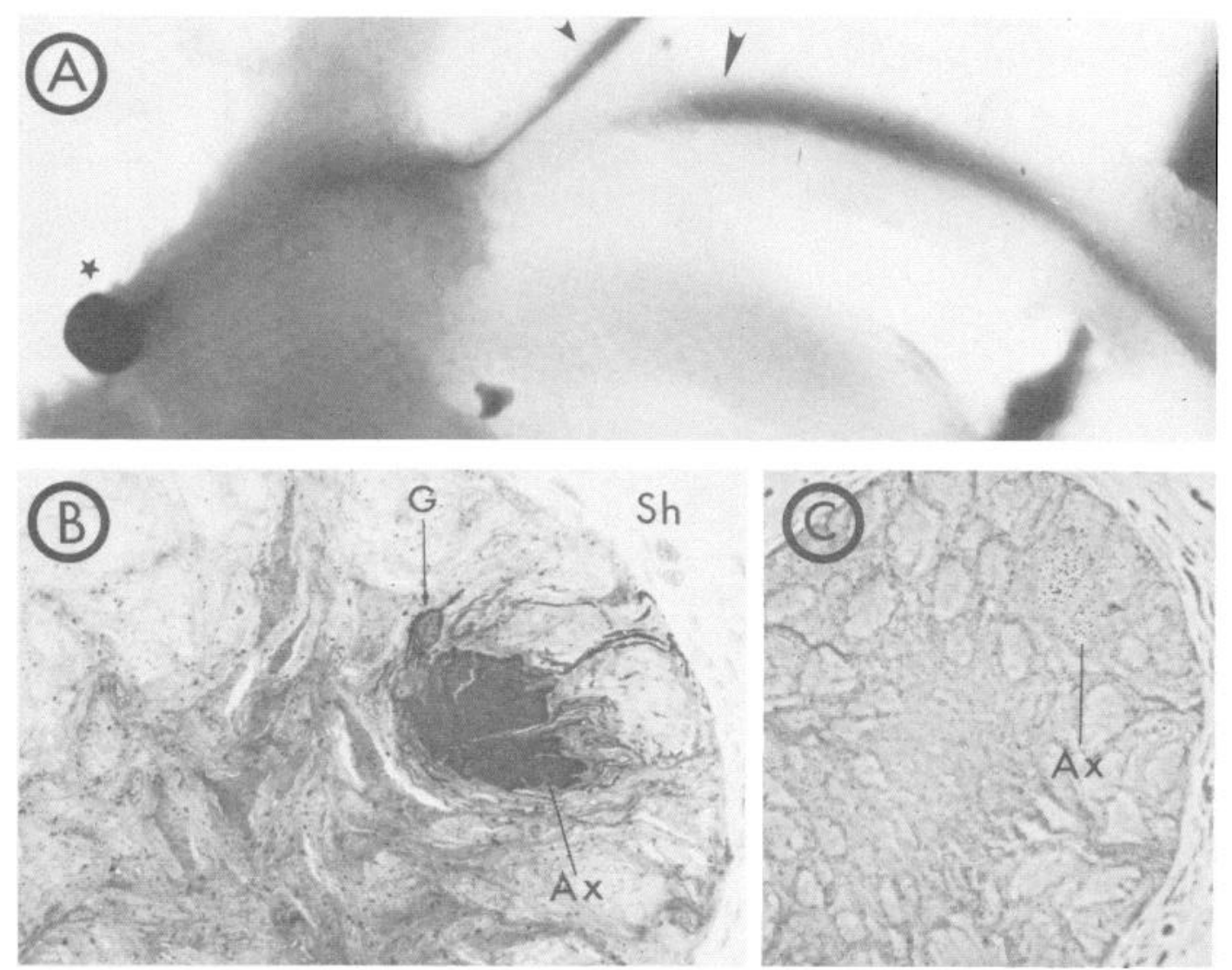

Figure 1. Distribution of reaction product $20 \mathrm{hr}$ after intrasomatic injection of HRP into a GCN. $A$, A photomicrograph of a whole mount of the cerebral ganglion. The cell body of the GCN (star) and initial segment of the axon are sharply defined by reaction product, but the main axon is obscured by the sheath and surrounding neural elements of the ganglion. Within the ganglion, the axon bifurcates, and the two branches can be seen emerging in the lip nerve (small arrowhead) and cerebrobuccal connective (large arrowhead). Reaction product in the two nerves does not delimit the axon branches sharply. Magnification $\times 90$. B, A photomicrograph of a 1- $\mu \mathrm{m}$ section of the connective cut in crosssection at approximately the point of the large arrowhead in $A$. The GCN's axon $(A x)$ is filled with reaction product as are the glial processes in its vicinity. This section is lightly stained with toluidine blue, revealing the nucleus of a glial cell $(G)$ with perinuclear cytoplasm and processes filled with HRP. A connective tissue sheath $(S h)$ surrounds the neural components of the nerve. Magnification $\times 950$. $C$, A phase contrast autoradiograph of a $2-\mu \mathrm{m}$ cross-section of a cerebrobuccal connective containing the axon of a GCN injected with $\left[{ }^{3} \mathrm{H}\right]$ fucose and treated for peroxidase activity. No reaction product is observed in the connective. The GCN's axon is identified by silver grains $(A x)$. Magnification $\times 950$.

nents. Light microscopic sections of the ganglion showed homogeneous filling of the GCN (Fig. $4 A$ ) but no label in any surrounding structures, which primarily were glial. Glial cells invaginate and interdigitate into the trophospongium of the cell near the axon hillock, and the cell body is cushioned by many glial cells that fill the spaces between it and other nerve cell bodies (see below).

Electron microscopic examination of the injected cell bodies and their initial segments showed that these regions of the neuron also were filled homogeneously with HRP, but areas of glial invagination and the surrounding glial cells were devoid of the label (Fig. 4, $B$ and $C$ ).

Subcellular distribution of HRP after uptake from the extracellular space. We think that it is unlikely that HRP is transferred to glial cells in the connective from the extracellular space in the ganglion. In all of these experiments, the connective tissue sheath was removed from the cerebral ganglion in the region containing the cell body of the neurons to be injected. Consequently, any enzyme that might have escaped during the injection should have been lost in the bath. Moreover, even if some HRP had diffused extracellularly from the site of the injection into the connective at a distance of $3 \mathrm{~mm}$, we would have expected it to be taken up by endocytosis. To determine whether glial cells in Aplysia can take up extracellular proteins, HRP was introduced into the extracellular space of the cerebrobuccal connective (see "Materials and Methods"). We found that glial cells in the cerebrobuccal connective took up HRP into internal membranous structures, vesicles, and endoplasmic reticulum (Fig. 5). This distribution of the label is unlike the homogeneous cytoplasmic staining that we observed after intraneuronal injection but is similar to the distributions resulting from endocytosis in other animals (Brightman, 1965; Holtzman et al., 1970; see Reineke, 1976, for other references). 


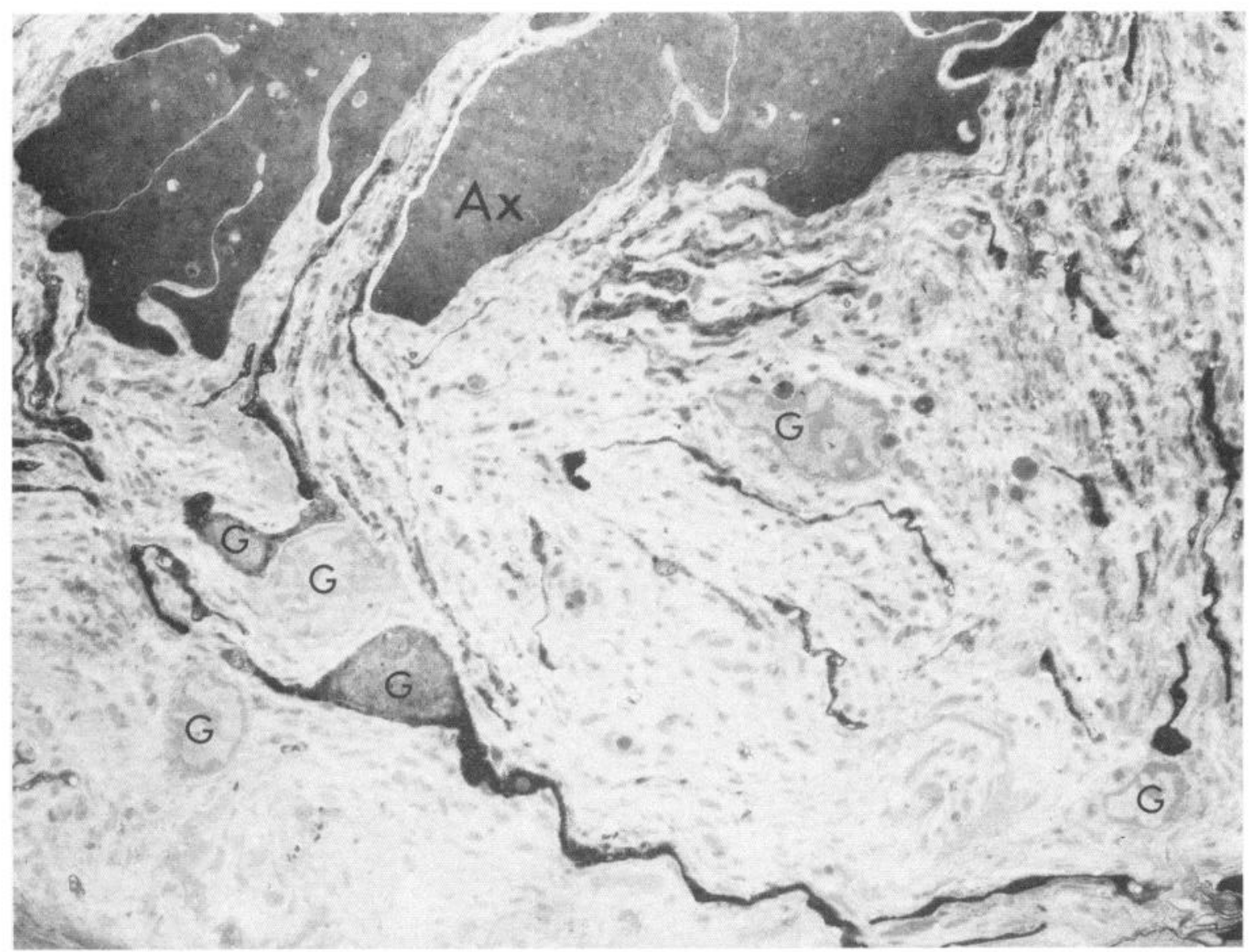

Figure 2. An unstained low magnification electron micrograph showing a GCN's axon $(A x)$ and nearby structures in the cerebrobuccal connective after intrasomatic injection of HRP. Labeled and unlabeled glial cell bodies $(G)$ are seen as well as labeled glial processes. Magnification $\times 4,200$.

Morphology of labeled and unlabeled glial cells. The absence of labeled glial cells in the ganglion after intrasomatic injection of the GCN and the presence of label only in some glial cells in the connective that are close to the axon of the injected neuron raise the question of whether there are functional differences between various types of glia. In the nerve, the relation of glia to axons varies according to the size of the axon (Fig. 6). As described by Coggeshall (1967) for the pleuroabdominal connective, we found that, in the cerebrobuccal connective, the smallest axons appear in bundles and have rounded profiles in cross-section. Each bundle is surrounded by one or two glial processes. Medium size axons are somewhat irregular in shape and are surrounded by a few layers of glial cell processes. The largest axons, of which the GCN's is one, are highly invaginated and are surrounded by several layers of glial processes that wrap around each axon circumferentially. Despite their relationship to axons, all glial cells in the connective appear to be similar ultrastructurally (Fig. 7, $D$ and $E$ ). Glial nuclei have a characteristic heterochromatic rim to the chromatin and often contain a nucleolus. They are surrounded by a thin layer of cytoplasm which contains Golgi cells, rough endoplasmic reticulum, glycogen granules, and lipid-containing lysosome-like bodies as well as occasional mitochondria. Desmosomes often connect glial processes to one another and to glial cell bodies.
The processes, which characteristically contain bundles of filaments, some glycogen, occasional mitochondria, and the same type of lipid granules as do the somata, run primarily within the cross-sectional plane, perpendicular to the longitudinal axis of the axon. This permits them to be distinguished from small axons, which are always cut in cross-section. The cytoplasmic ground substance of the glia usually is somewhat darker than that of axonal profiles. The glial processes often cause the larger axons to appear lobulated, sometimes almost cutting them apart.

We have not been able to find any morphological characteristics that would allow us to distinguish those glial cells that are filled with HRP after intracellular injection of the GCN from the other, unlabeled glial cells in the cerebrobuccal connective (Figs. 3, 4, 6, and 7). The relationships of the labeled and unlabeled cells and processes to the GCN's axon are similar; both wrap around the axon within the plane of cross-section. Some glial cell bodies and processes close to the GCN's axon are not labeled, while other more distant cells and processes are. We also find that, of the many glial processes which actually invaginate into the axon, some are labeled and some are not. Organelles that are not obscured by reaction product (mitochondria, nuclei, gliosomes, and desmosomes) appear similar in both labeled and unlabeled glia. The only morphological distinction between labeled 

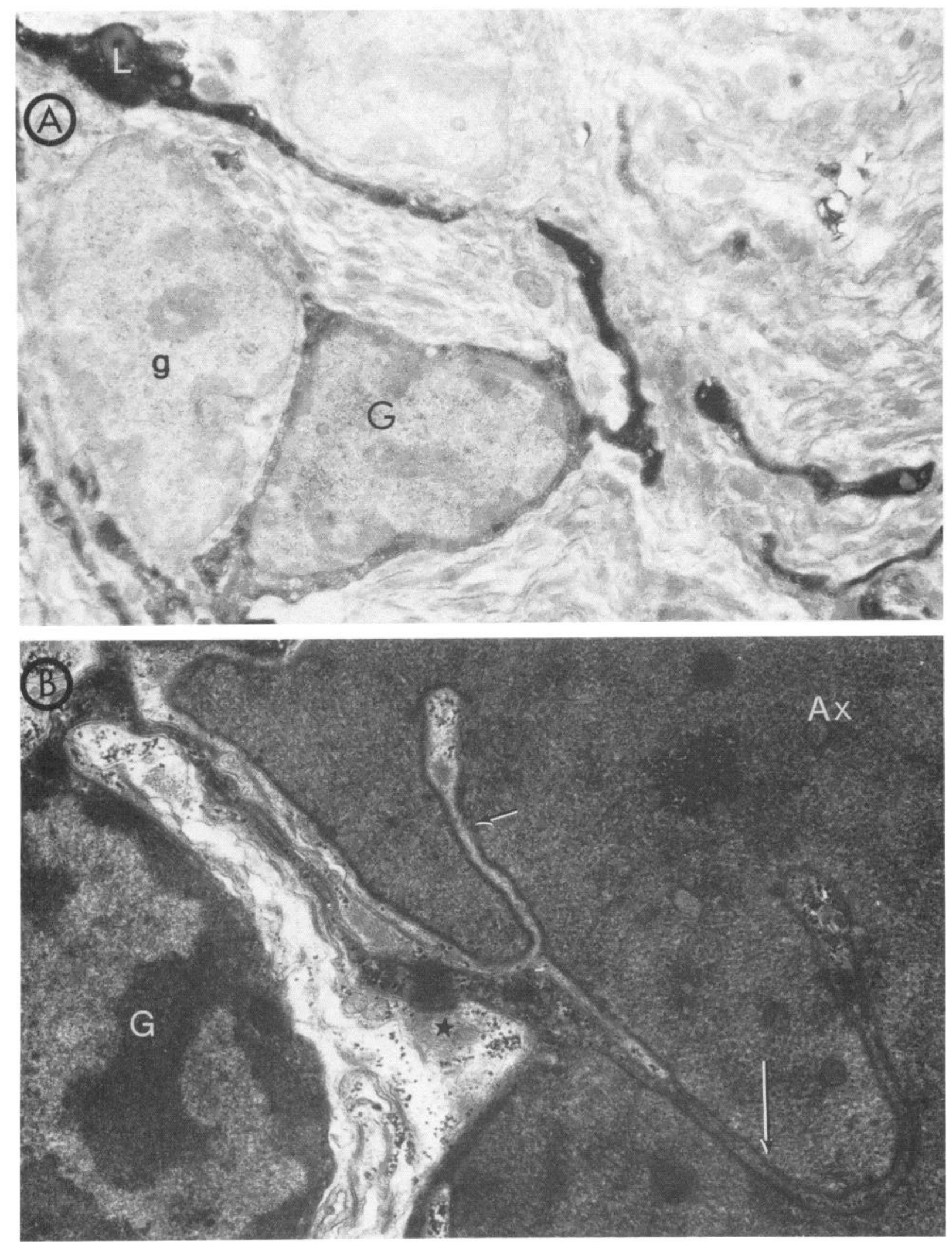

Figure 3. High magnification views of labeled glial cells near a GCN's axon in the cerebrobuccal connective. In $A$, a glial cell (G) $10 \mu \mathrm{m}$ away from the GCN's axon has a thin rim of perinuclear cytoplasm, a small lysosome $(L)$, and a nucleus with a heterochromatic rim. Another glial cell $(g)$ does not contain reaction product. Magnification $\times 14,200$. B, shows a labeled glial cell body close to the GCN's axon $(A x)$. A labeled and an unlabeled process are joined by a desmosome (star) from which filaments extend. Both labeled (large arrow) and an unlabeled (small arrow) processes invaginate the axon. Magnification $\times 25,300$. 

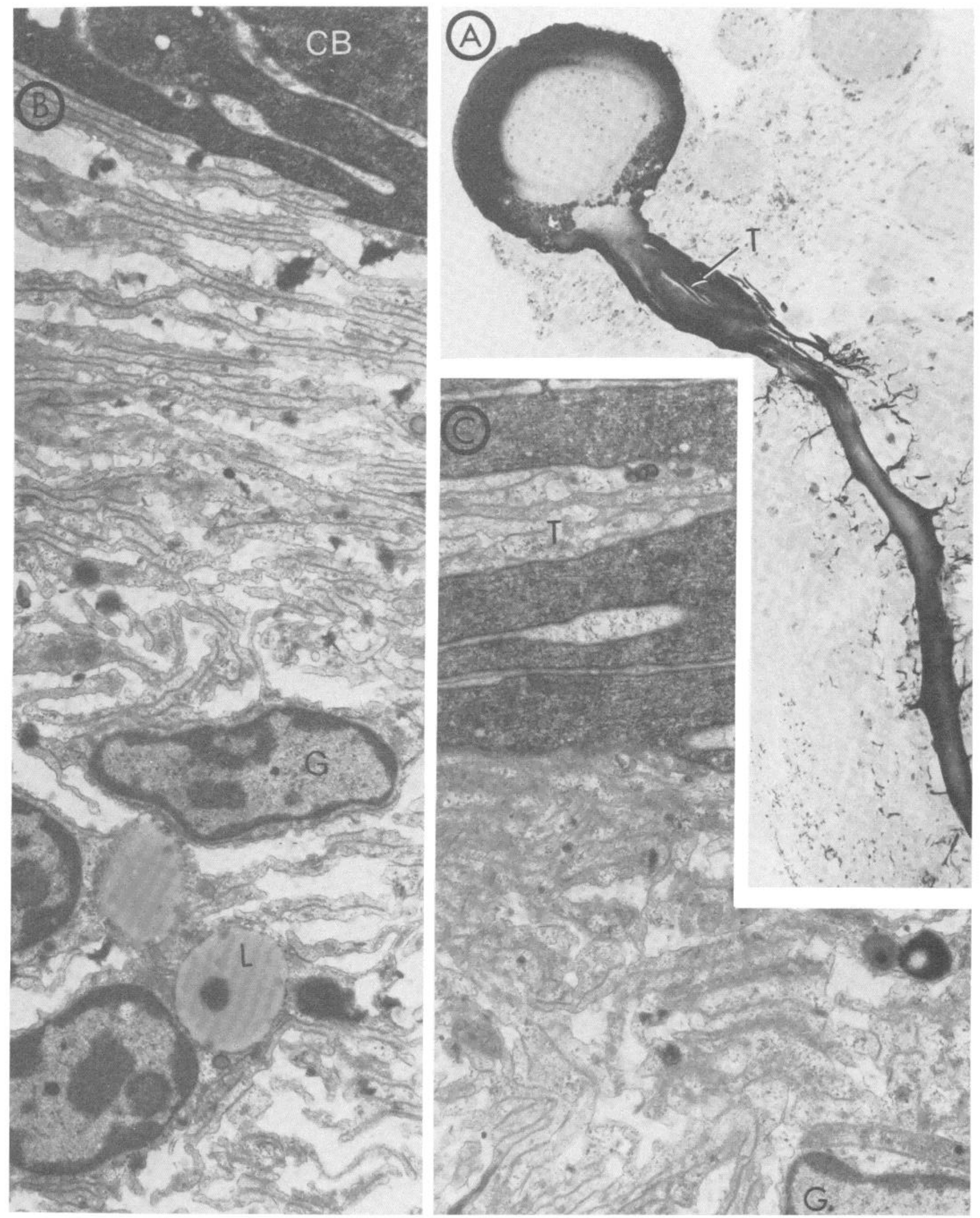

Figure 4. Views of the cell body and proximal axon $20 \mathrm{hr}$ after injection with HRP. A, A photomicrograph of a 4- $\mu \mathrm{m}$ section containing the cell body and main axon. No reaction product is seen in neighboring neurons or glia. The area of the trophospongium $(T)$ also lacks reaction product. Many short dendritic tufts branch off from the main axon into the neuropil. The section is stained with toluidine blue. Magnification $\times 320$. B An electron micrograph of the cell body $(C B)$ and neighboring structures. The cell body is filled with reaction product. Glial cells $(G)$ containing large lysosomes $(L)$ and glial processes are loosely packed near the cell body and are not labeled. Magnification $\times 9,200$. $C$, shows that the extensive glial interdigitations and surrounding glial processes also are not labeled. Magnification $\times 9,200$. 

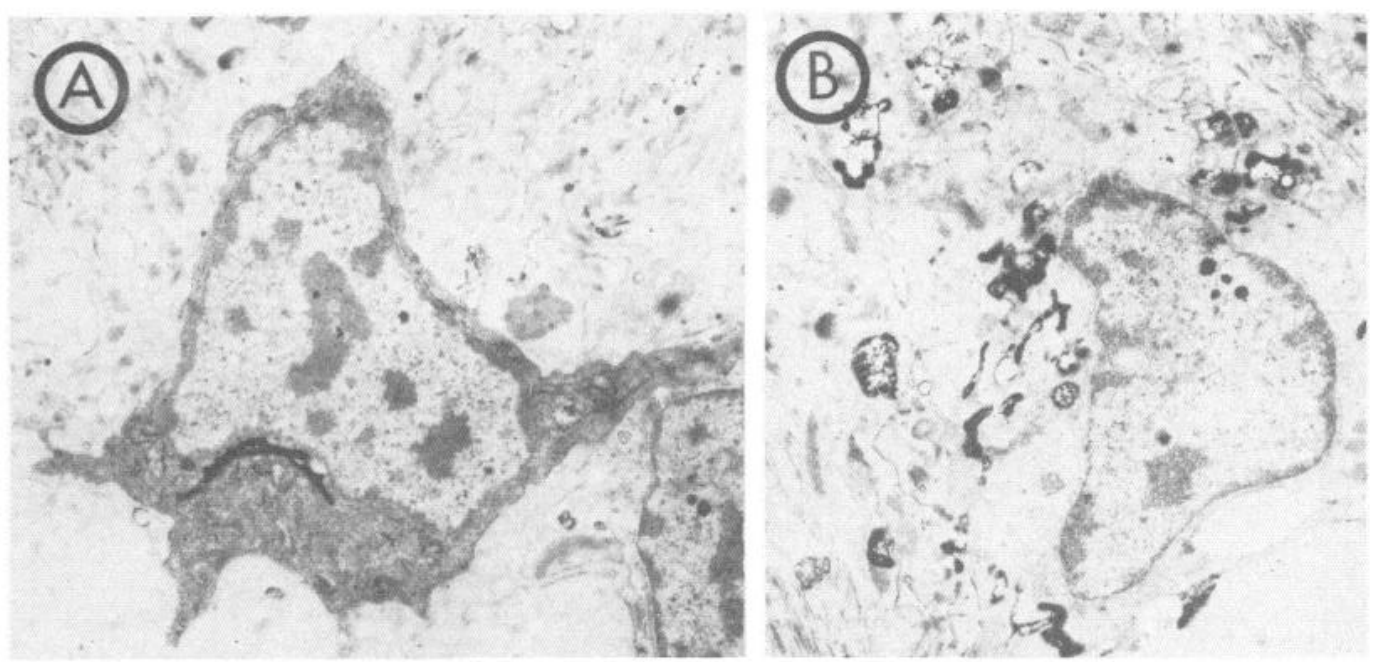

Figure 5. Electron micrographs of glial cells from the connective near the buccal ganglion after exposure to extracellular HRP (see "Materials and Methods"). Presumably, HRP has been taken up by endocytosis, and membranous organelles, such as the Golgi cells in $A$ and the vesicular structures in $B$, contain reaction product. The cytoplasm is not, however, stained diffusely as in Figures 2 and 3 . Magnification $\times 14,300$.

and unlabeled glia in the connective is that labeled cells or processes do not occur at a distance of more than about $20 \mu \mathrm{m}$ away from the axon.

In the ganglia, glial cells are most prevalent in the cell body layer and around the large axonal trunks that arise from cell bodies (Fig. 7, $A$ and $B$ ). Glial cells also fill the spaces of the subcapsular sinus (Rosenbluth, 1963; Colonnier et al., 1979). The glial cells in the ganglion differ morphologically from those in the connective in several ways. They have larger ovoid nuclei with more extensive perinuclear cytoplasm and contain large lysosomes 2 or 3 times the size of those in the glial cells in the connective. We did not see any desmosomes between the processes, which do not contain many filaments. Also, contacts with the morphological appearances of synapses were observed on glial cells in ganglia (Colonnier et al., 1979; Schwartz and Shkolnik, 1981), but we saw none in the connective.

\section{Discussion}

After injecting the cell body of the GCN, we found that HRP labeled some glial cells along the entire length of the two nerves that contain axon branches of the neuron, starting at a distance of about $3 \mathrm{~mm}$ from the site of the injection. All of the labeled glia were situated within 20 $\mu \mathrm{m}$ (1 to 1.5 axon diameters) of the GCN's axons; no cells outside of this region were labeled. Not all glial cells close to the axon of the GCN contained HRP, however, suggesting that proximity alone is insufficient to explain the labeling. This idea is strengthened further because glial cells within the cerebral ganglion, which come into intimate contact with the cell body and initial segment of the axon, did not contain any HRP reaction product. Most striking was the absence of labeled glia in the area of the axon hillock called the trophospongium, where glial fingers penetrate through the neuron, forming a reticulum of glial processes. This selectivity of labeling confirms that adaxonal glial cells differ from glia in the ganglion, an idea that is suggested by the differences in morphology between the glial cells in the two regions that Coggeshall (1967) originally described and that we have extended here.

In addition to the differences between glial cells in ganglion and nerve, is it useful to think of the differences between glia within the nerve? Our observation that, for a glial cell to be labeled, it must be situated close to an axon of the injected neuron but that, at most, only a third of the adaxonal glial cells are labeled suggests that the labeled glial cells have some special connection with the GCN's axon. It is conceivable that we might have failed to detect the presence of HRP in some adaxonal glial cells because of the variability or insensitivity of our histochemical procedures. We find it attractive to think, however, that the selective pattern of labeling observed results from glial specialization, because glial cells already are known to interact specifically with specific neurons. Interactions between specific glia and specific neurons during development are also well known. An example is the guidance provided to granule cells by Bergmann glia in the developing cerebellum (see Sidman and Rakic, 1973 , for review). The mechanisms underlying glial specialization have not yet been characterized, but the ability to identify glial cells specialized for individual neurons may provide the opportunity to determine how a neuron relates specifically to a group of glial cells.

Mechanism of transfer. We found no labeled cells within the ganglion close to the site of the injection. We therefore think that it is unlikely that HRP traveled from the site of the injection to glial cells in the nerve by any route other than through the injected neuron. Even though general uptake from the bath can occur, if this were the mechanism of transfer, we might expect the glial cells nearest the site of the injection to be heavily labeled and the distribution of HRP within the labeled cells not to be diffuse in the cytoplasm. Labeling glia by uptake presumably occurs by endocytosis, since the marker is localized to vesicles and short tubules and is not distributed diffusely throughout the cytoplasm. The diffuse intracytoplasmic localization that we observed after intraneuronal injection also strongly argues against 


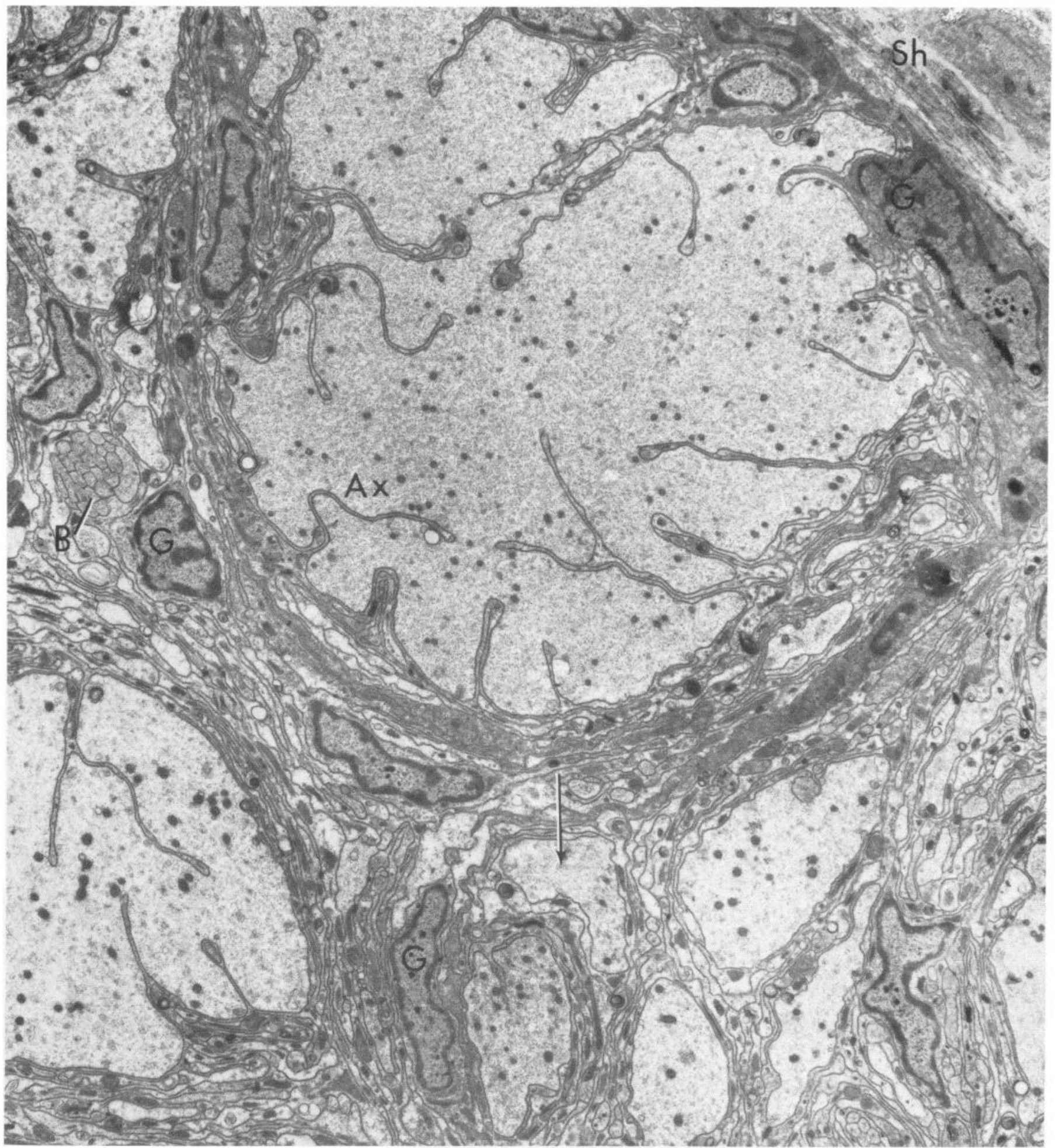

Figure 6. A low magnification electron micrograph showing the relationships of glia $(G)$ to axons $(A x)$ in the cerebrobuccal connective. The GCN's giant axon is thickly invested by glial processes and is deeply invaginated by them, giving it a lobulated appearance. Medium size axons (arrows) have only a few layers of processes and are not lobulated. Many small axons in bundles $(B)$ are surrounded by a single process, but most of the axons do not contact glia at all. Sh, Connective tissue sheath. Magnification $\times 6,100$.

Figure 7. Differences between glial cells in the ganglion and in the connective. In the ganglion $(A$ and $B)$, glial cells contact neuronal cell bodies $(N)$; some others $(C)$ form end-feet on the basal lamina of the sheath $(S h)$ and lie in the subcapsular sinus $(S)$. Glial cells in the ganglion contain large lysosomes $(L)$ in their extensive perinuclear cytoplasm. Glial cells in the connective $(D$ and $E)$ are smaller and have a thin rim of cytoplasm around their nuclei. They form desmosomes (star) with one another and contain small lysosomes and many bundles of filaments. Medium axons are seen at the arrows. The sections shown in $A, B$, and $D$ are fixed in $3 \%$ glutaraldehyde, $3 \%$ formaldehyde and those in $C$ and $E$ is fixed in $6 \%$ glutaraldehyde. All except $E$ are stained en bloc with uranyl acetate. Magnification $\times 11,700$. 

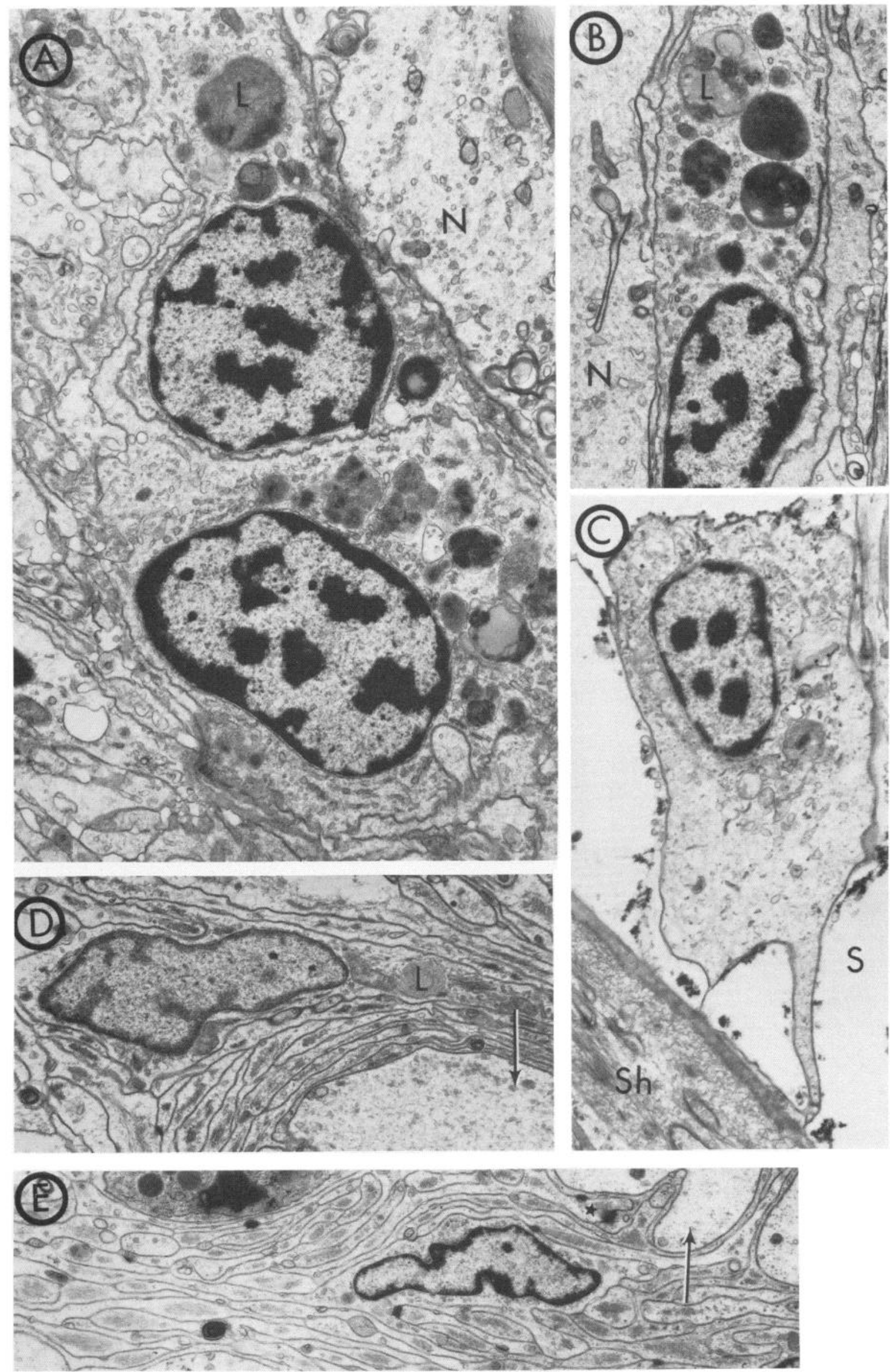
the idea that HRP was transferred by exocytosis followed by endocytosis as suggested for the intracellular movement of materials between axon and glia in squid and crayfish (Lasek et al., 1976; Viancour et al., 1981) because both exocytosis and endocytosis involve membranous organelles.

Preliminary experiments indicate that labeling of the adaxonal glia of Aplysia giant neurons is not restricted to the giant serotonergic neuron. When HRP was injected into $R 2$, the giant cholinergic neuron of the abdominal ganglion, glial cells associated with R2's major axon in the right connective were labeled selectively. Perhaps other giant neurons each have their own set of specific glial cells.

We presume that the transfer of HRP reflects a functional connection between the axon of the GCN and associated glial cells that permit passage of the $M_{\mathrm{r}}=$ 40,000 protein. There are several possible mechanisms for the transfer. One likely mechanism is a specialized junction connecting the axoplasm of the GCN with the cytoplasm of a specific set of glial cells. Gap junctions would not be large enough to permit passage of a molecule the size of HRP. Recently, Perrachia (1981) has reported junctions in crayfish nerves that would allow proteins with molecular weights of up to 200,000 to pass from neuron to glia. Another instance of interconnections between cells occurs in Drosophila oocytes, each of which are connected in a specific manner to nurse cells by cytoplasmic bridges that are large enough to permit passage of centrioles (Mahowald and Strassheim, 1970). In the GCN, however, it appears that only soluble cytoplasmic constituents can be transferred, since neither serotonergic vesicles (Goldman et al., 1976) nor other membranous organelles labeled after intrasomatic injection of ${ }^{3} \mathrm{H}$-labeled sugar precursors of membrane glycoproteins (Ambron et al., 1980; Schwartz and Shkolnik, 1981) were observed in glial cells near the GCN's axon nor near axons of any other identified Aplysia neuron (Thompson et al., 1976). We have not seen any structures that might act as junctions along Aplysia axons, but they may be rare or not obvious in conventional transmission electron microscopy. Alternatively, HRP may be transferred through membranes because of specific receptors for the protein in the axolemma. This mechanism would require the transfer process to be selective for some structural feature of the HRP molecule, for example, sugar residues or signal sequences (see Sabatini et al., 1982), and could be probed by testing whether other proteins also can be transferred.

Because of observations in the septate axon of the crayfish and in reaggregated blastomeres of Fundulus, Bennett (1973) has suggested that, after fixation, some HRP can cross through cell membranes that were coupled electrotonically in the living cell and that fixation might cause breaks in the junctional membrane. Although there is little other evidence from earlier studies, either in Aplysia or in many other animals, that any significant amount of HRP moves through membranes as an artifact of fixation, it is difficult to prove directly that HRP is transferred to glia from the living giant neuron. Unfortunately, techniques for cytochemical localization of the enzyme in unfixed tissue have not yet been developed in Aplysia. Nevertheless, several lines of evidence argue against the idea that the appearance of HRP in glia that we have observed is a consequence of fixation. Thus, when HRP was injected into the GCN (this paper) or into other identified neurons (see, for example, Bailey et al., 1979), the enzyme did not appear in glia within the ganglion that contained the injected cell body. If the tracer moves during fixation, we might expect transfer to take place within the ganglion close to the site of the injection where the concentration of HRP is highest as well as in the more distant nerves. HRP administered extracellularly and taken up endocytotically also might be expected to move artifactually, but the enzyme has been found to be sharply localized both in Aplysia (Fig. 5) and in countless other animals (see, for example, Keefer, 1978). Finally, failure to observe the transfer of other substances that have been injected into the GCN provides some indication that fixation does not result in the gross redistribution of axonal constituents: $\left[{ }^{3} \mathrm{H}\right]$ serotonin (Goldman et al., 1976), glycoproteins labeled with ${ }^{3} \mathrm{H}$-labeled sugar precursors, some of which are soluble (Ambron et al., 1980; Cleary and Schwartz, 1982), myoglobin, and HRP conjugated to the lectin, wheat germ agglutinin, did not label adaxonal glial cells.

Determining how glial cells become labeled by HRP is important because the mechanism could provide insight into the physiological role of the transfer. Most important would be to determine which of the many functions commonly attributed to glia-developmental, trophic, buffering, scavenger, or supporting-is served by the intercellular transfer of marcomolecules. A structurally open channel would imply that axons and their associated glial cells are coupled electrically. Glial cells are coupled electrically to other glia through gap junctions (Orkand, 1977; Gutnik et al., 1981). Are glial cells that are associated with one axon coupled to glia associated with other axons or can one glial cell be associated with more than one specific axon? If so, in addition to their supporting functions, glial cells also may play a role in intercellular signaling.

\section{References}

Ambron, R. 'T., J. E. Goldman, L. J. Shkolnik, and J. H. Schwartz (1980) Synthesis and axonal transport of membrane glycoproteins in an identified serotonergic neuron of Aplysia. J. Neurophysiol. 43: 929-944.

Bailey, C. H., E. B. Thompson, V. F. Castellucci, and E. R. Kandel (1979) Ultrastructure of the synapses of sensory neurons that mediate the gill-withdrawal reflex in Aplysia. J. Neurocytol. 8: 415-444.

Bennett, M. V. L. (1973) Permeability and structure of electrotonic junctions and intercellular movements of tracers. In Intercellular Staining in Neurobiology, S. B. Kater and C. Nicholson, eds., pp. 115-134, Springer-Verlag, New York.

Brightman, M. W. (1965) The distribution within the brain of ferritin injected into the cerebrospinal fluid compartments. II. Parenchyma distribution. Am. J. Anat. 117: 193-220.

Cleary, I. .J., and J. H. Schwartz (1982) Identification of the principal organelle carried by fast axonal transport in the giant cerebral neuron of Aplysia. Soc. Neurosci. Abstr. 8: 828.

Coggeshall, R. E. (1967) A light and electron microscope study of the abdominal ganglion of Aplysia californica. J. Neurophysiol. 30: 1263-1287. 
Coggeshall, R. E., and D. W. Fawcett (1964) The fine structure of the central nervous system of the leech Hirudo medicinalis. J. Neurophysiol. 27: 229-289.

Colonnier, M., J. P. Tremblay, and H. McLennon (1979) Synaptic contacts on glial cells in the abdominal ganglion of Aplysia californica. J. Comp. Neurol. 188: 391-400.

Eisenstadt, M., J. E. Goldman, E. R. Kandel, H. Koike, J. Koester, and J. H. Schwartz (1973) Intrasomatic injection of radioactive precursors for studying transmitter synthesis in identified neurons of Aplysia californica. Proc. Natl. Acad. Sci. U. S. A. 70: 3371-3375.

Gainer, H., L. Tasaki, and R. J. Lasek (1977) Evidence for the glia-neuron protein transfer hypothesis from intracellular perfusion studies of squid giant axons. J. Cell Biol. 74: 524-530.

Goldman, S. E., K. S. Kim, and J. H. Schwartz (1976) Axonal transport of ${ }^{3} \mathrm{H}$-serotonin in an identified neuron of Aplysia californica. J. Cell Biol. 70: 304-318.

Goldstein, R. S., K. R. Weiss, and J. H. Schwartz (1982) Intraneuronal injection of horseradish peroxidase labels glial cells associated with an identified Aplysia neuron. Soc. Neurosci. Abstr. 8: 715.

Gutnick, M. J., B. W. Conners, and B. R. Ransom (1981) Dye coupling between glial cells in the guinea pig neocortical slice. Brain Res. 213: 486-492.

Holtzman, E., A. R. Freeman, and L. A. Kashner (1970) A cytochemical and electron microscope study of channels in the Schwann cells surrounding lobster giant axons. J. Cell Biol. 44: 438-445.

Kandel, E. R. (1976) Cellular Basis of Behavior, Freeman, San Francisco.

Keefer, D. A. (1978) Horseradish peroxidase as a retrogradely transported, detailed dendritic marker. Brain Res. 140: 15-32.

Kuffler, S. W., and J. G. Nicholls (1966) The physiology of neuroglial cells. Ergeb. Physiol. Biol. Chem. Exp. Pharmakol. 57: 1-90.

Lasek, R. J., H. Gainer, and J. L. Barker (1977) Cell to cell transfer of glial proteins to the squid giant axon. The glianeuron protein transfer hypothesis. J. Cell Biol. 74: 501-523.

Mahowald, A., and J. Strassheim (1970) Intercellular migration of centrioles in the germarium of Drosophila melanogaster J. Cell Biol. 45: 306-320.

Muller, K. J., and O. J. McMahan (1976) The shapes of sensory and motor neurons and the distribution of their synapses in ganglia of the leech: A study using intracellular injection of horseradish peroxidase. Proc. R. Soc. Lond. (Biol.) 194: 481-499.

Orkand, R. E. (1977) Glial cells. In Handbook of Physiology. Vol. I: Cellular Biology of Neurons, Part II, pp. 855-875, American Physiological Society, Bethesda, MD.

Perrachia, C. (1981) Direct communication between axons and sheath cells in crayfish. Nature 290: 597-598.

Reineke, M. (1976) The glial cells of the cerebral ganglia of Helix pomatia L. II. Uptake of ferritin and ${ }^{3} \mathrm{H}$-glutamate. Cell Tissue Res. 169: 361-382.

Rosenbluth, J. (1963) The visceral ganglion of Aplysia californica. Z. Zellforsch. Mikrosk. Anat. 60: 213-236.

Sabatini, D. D., G. Dreibich, T. Morimoto, and M. Adesnick (1982) Mechanisms of incorporation of proteins in membrancs and organelles. J. Cell Biol. 92: 1-22.

Schwartz, J. H., and L. J. Shkolnik (1981) The giant serotonergic neuron of Aplysia: A multi-targeted nerve cell. J. Neurosci. 1: 606-619.

Sidman, R. L., and P. Rakic (1973) Neuronal migration, with special reference to developing human brain: A review. Brain Res. 62: 1-35.

Thompson, E. B., J. H. Schwartz, and E. R. Kandel (1976) A radioautographic analysis in the light and electron microscope of identified Aplysia neurons and their processes after intrasomatic injection of $\left({ }^{2} \mathrm{H}\right)$-L-fucose. Brain Res. 112: 251-281.

Varon, S. S., and G. G. Somjen (1979) Neuron-glia interactions. Neurosci. Res. Program Bull. 17: 95-130.

Viancour, T. A., G. P. Bittner, and M. L. Balligner (1981) Selective transfer of Lucifer Yellow $\mathrm{CH}$ from axoplasm to adaxonal glia. Nature 239: 65-67.

Weiss, K. R., J. L. Cohen, and I. Kupfermann (1978) Modulatory control of buccal musculature by a serotonergic neuron (metacerebral cell) in Aplysia. J. Neurophysiol. 41: 181-203. 